and other residues. They also recommend that cleaning items should be disposable or thoroughly cleaned and disinfected or sterilized between uses. ${ }^{8}$

This study has several limitations. Our manually soiled PTFE tubes did not contain a biofilm in the sense of Michelle Alfa's definition. ${ }^{9}$ The "buildup biofilm" in endoscopes develops as a result of cyclical exposure to wet and dry phases during use and reprocessing. Because certain cleaning devices may compound the accretion of residual soil by causing surface abrasion or grooving of the lumen wall, additional research to address this issue could yield helpful insights. ${ }^{7}$ Furthermore, the conclusion of our study is not necessarily generalizable for several reasons. Experiments were only performed at a single hospital site by 1 person, and only endoscope models were used. A future larger study could include different types of endoscopes from different manufacturers in a real-life hospital setting.

Acknowledgments. Sofie Colman performed the calculations and wrote the manuscript. All authors discussed the results and commented on the manuscript.

Financial support. No financial support was provided relevant to this article.

Conflicts of interest. All authors report no conflicts of interest relevant to this article.

Supplementary material. To view supplementary material for this article, please visit https://doi.org/10.1017/ice.2021.170

\section{References}

1. Kovaleva J, Peters FT, van der Mei HC, Degener JE. Transmission of infection by flexible gastrointestinal endoscopy and bronchoscopy. Clin Microbiol Rev 2013;26:231-254.

2. Agentschap Zorg en Gezondheid. Eisenkader high level desinfectie van flexibele endoscopen in het ziekenhuis. 2014.

3. Garces E. Consequences of improper scope cleaning. Infection Control Today March 1, 2008. https://www.infectioncontroltoday.com/view/scopecleaning-and-repair

4. Cattoir L, Vanzieleghem T, Florin L, et al. Surveillance of endoscopes: comparison of different sampling techniques. Infect Control Hosp Epidemiol 2017;38:1062-1069.

5. Batailler P, Saviuc P, Picot-Gueraud R, Bosson JL, Mallaret MR. Usefulness of adenosinetriphosphate bioluminescence assay (ATPmetry) for monitoring the reprocessing of endoscopes. Infect Control Hosp Epidemiol 2015;36: 1437-1443.

6. Charlton TS. A comparison of the efficacy of lumen-cleaning devices for flexible gastrointestinal endoscopes. Aust Infect Control 2007;12:81-90.

7. Beilenhoff U, Neumann CS, Rey JF, Biering H, Blum R, Schmidt V. ESGEESGENA guideline for quality assurance in reprocessing: microbiological surveillance testing in endoscopy. Endoscopy 2007;39:175-181.

8. Petersen BT, Chennat J, Cohen J, et al. Multisociety guideline on reprocessing flexible gastrointestinal endoscopes: 2011. Gastrointest Endosc 2011;73: $1075-1084$.

9. Alfa MJ, Howie R. Modeling microbial survival in buildup biofilm for complex medical devices. BMC Infect Dis 2009;9:56-56.

\title{
Evaluation of Escherichia coli resistance to fluoroquinolones in men undergoing prostate procedures: Is it time to change preoperative prophylaxis?
}

\author{
Colin D. Sperling $M D^{1}$, Lucia Rose PharmD ${ }^{2,3}$, Hailiu Yang $M D^{4}$, Dana D. Byrne $M D^{3}$, Henry S. Fraimow $\mathrm{MD}^{3}$, \\ Jeffrey J. Tomaszewski MD ${ }^{5}$ and Allen D. Seftel MD \\ ${ }^{1}$ Department of Urology, Hospital of the University of Pennsylvania, Philadelphia, Pennsylvania, ${ }^{2}$ Department of Pharmacy, Cooper University Hospital, Camden, \\ New Jersey, ${ }^{3}$ Division of Infectious Diseases, Department of Medicine, Cooper University Hospital, Camden, New Jersey, ${ }^{4}$ Gould Medical Group, Division of \\ Urology, Modesto, California, United States and ${ }^{5}$ Division of Urology, Department of Surgery, Cooper University Hospital, Camden, New Jersey
}

To the Editor-Urinary tract infections and prostatitis account for most postoperative infectious complications following prostate procedures, by direct inoculation of bacteria from skin or rectal mucosa into the prostate or urinary tract. Escherichia coli is the most common pathogen in $75 \%-90 \%$ of cases. ${ }^{1}$ Rates of sepsis from a urinary source are also rising, which may be secondary to increasing prevalence of antibiotic resistant uropathogens. ${ }^{2}$ Although the mechanisms leading to antibiotic resistance are complex, inappropriate utilization of antibiotics, particularly fluoroquinolones (FQs), has been shown to correlate with postprocedural infection risk due to ease of bacterial acquisition of resistance

\footnotetext{
Author for correspondence: Lucia Rose, E-mail: lucia.rose84@gmail.com; Rose-lucia@ cooperhealth.edu

Cite this article: Sperling CD, et al. (2022). Evaluation of Escherichia coli resistance to fluoroquinolones in men undergoing prostate procedures: Is it time to change preoperative prophylaxis?. Infection Control \& Hospital Epidemiology, 43: 1078-1080, https://doi.org/ 10.1017/ice.2021.168
}

gene mutations. ${ }^{3}$ The Infectious Diseases Society of America (IDSA) now recommends avoiding empiric FQ use in genitourinary infections unless regional antibiogram data shows $<20 \%$ FQ resistance to $E$. coli.

Previous studies have demonstrated an increasing prevalence of FQ-resistant E. coli in the rectal vault of men undergoing transrectal ultrasound-guided prostate biopsy (TRUS-PBx). ${ }^{4}$ Despite these trends, the most current American Urological Association (AUA) best-practice statement, last amended in 2014, still recommends FQs as primary antimicrobial agents for preoperative prophylaxis for TRUS-PBx and transurethral procedures. ${ }^{5}$

Fluoroquinolone resistance to E. coli has reached $50 \%$ in some regions of the United States. ${ }^{6}$ As of 2018, our institution had been using ciprofloxacin preoperatively due to a lack of data supporting alternative agents. Our institution-wide antibiogram reports $\sim 30 \%$ FQ resistance to $E$. coli, but we were unsure of the generalizability to men undergoing prostate procedures. We aimed to evaluate FQ 


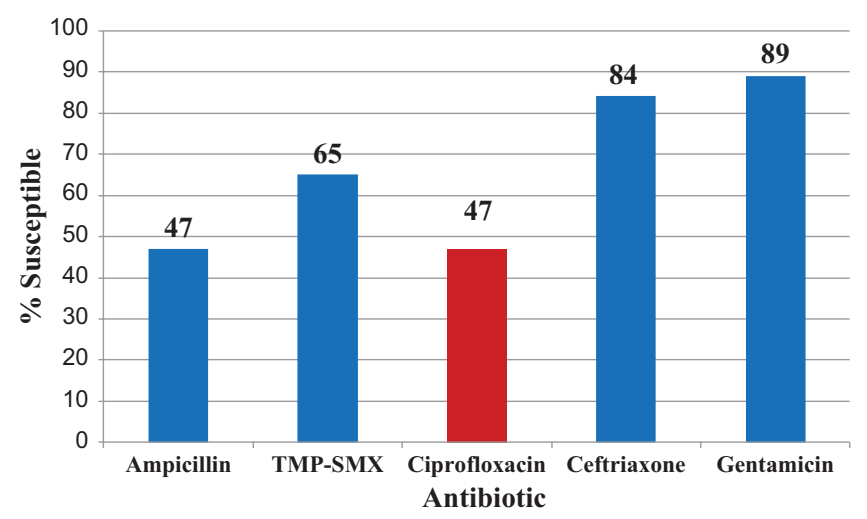

Fig. 1. Antibiotic susceptibility pattern of $E$. coli isolates to common preoperative antibiotics.

resistance among E. coli isolates in this population at Cooper University Hospital. We assessed E. coli susceptibility as a marker for the presence or absence of antibiotic-resistant GU flora.

\section{Methods}

We utilized TheraDoc (Premier Healthcare, Charlotte, NC) to identify men $\geq 18$ years of age who underwent a primary prostate procedure between January 2014 and December 2017 and had positive E. coli isolates from either urine or blood within the preceding 12 months. For men requiring multiple surgical procedures, only data prior to the first prostate procedure were included for analysis. Medical records were reviewed via EPIC (EPIC Systems, Verona, WI). The primary end point was the prevalence of FQ-resistant $E$. coli in men undergoing prostate procedures. This evaluation was approved by the institutional review board as a performance improvement project.

\section{Results}

In total, 57 men met criteria for chart evaluation. The most common procedure was radical prostatectomy (44\%), followed by prostate photovaporization (23\%), transurethral resection of the prostate $(21 \%)$, and TRUS-PBx (12\%). All patients received preoperative antibiotics and most received a single agent. Cefazolin were administered to $49 \%$ and FQs were administered to $26 \%$ of patients. Of $57 \mathrm{E}$. coli isolates, $31(53 \%)$ of 57 were FQ resistant whereas only $8(14 \%)$ of 57 were ceftriaxone resistant (Fig. 1). The $32 \%$ overall hospital rate of FQ-resistant E. coli was significantly lower than the $54 \%$ rate in our study population $(P=.0010)$. Overall, 41 patients $(72 \%)$ received FQs within 1 year of their procedure. Fluoroquinolone resistance was significantly associated with prior FQ use $(P=.0091)$. Of 8 patients with a $\beta$-lactam allergy, 6 received an FQ (75\%) compared to $14(29 \%)$ of 49 patients without allergy $(P=.011)$.

\section{Discussion}

The rate of FQ-resistant E. coli was very high in our population of men undergoing invasive urologic procedures. Our E. coli FQ resistance rate of $54 \%$ was considerably higher than the $32 \%$ rate predicted from our hospital antibiogram. These findings indicate that regional or hospital-wide antibiograms may not accurately reflect resistance in a subset of male urologic patients, and they underscore the need to tailor the selection of empirical prophylactic antibiotics to accommodate patient-specific factors. Although patients with available prior positive E. coli cultures may not be entirely representative of all men undergoing prostate procedures, both are groups with high risk of prior antibiotic and FQ exposures.

We also demonstrated that prior FQ use was associated with the presence of FQ-resistant isolates. In the era of prostate-specific antigen (PSA)-based prostate cancer screening, appropriate management of elevated PSA remains an intensely debated topic in the urological literature. Benign etiologies, such as prostatitis or benign prostatic hyperplasia, can result in PSA elevations. To avoid a potentially unnecessary prostate procedure, clinicians often prescribe antibiotics to treat a possible subclinical infection to determine whether PSA levels normalize. It is estimated that up to a third of nonurologist physicians still adhere to this practice despite the paucity of scientific evidence supporting it and the potential for unfavorable sequelae including antimicrobial resistance and a higher likelihood of post-TRUS-PBx sepsis. ${ }^{7,8}$ We believe that our findings of a direct association between prior FQ use and subsequent resistance is significant and adds to the growing body of data supporting utilization of alternative antibiotics for empirical prophylaxis and treatment.

In conclusion, FQ resistance to $E$. coli is unacceptably high $(53 \%)$ in this population of adult men undergoing prostate procedures. Whole-hospital antibiograms may not be reliable to predict $E$. coli resistance in patients undergoing urologic procedures. If preprocedure culture data are available, the choice of prophylaxis should be targeted toward the bacterial isolate and its corresponding susceptibilities. In the absence of preprocedural culture data, an alternative agent such as ceftriaxone should be considered for standard preoperative prophylaxis for transurethral or transrectal procedures. First-generation cephalosporins remain the first choice for radical prostatectomy.

\section{Acknowledgments.}

Financial support. No financial support was provided relevant to this article.

Conflicts of interest. All authors report no conflicts of interest relevant to this article.

\section{References}

1. Williamson DA, Barrett LK, Rogers BA, Freeman JT, Hadway P, Paterson DL. Infectious complications following transrectal ultrasound-guided prostate biopsy: new challenges in the era of multidrug-resistant Escherichia coli. Clin Infect Dis 2013;57:267-274.

2. Halpern JA, Sedrakyan A, Dinerman B, Hsu WC, Mao J, Hu JC. Indications, utilization and complications following prostate biopsy: New York state analysis. J Urol 2017;197:1020-1025.

3. Toner L, Bolton DM, Lawrentschuk N. Prevention of sepsis prior to prostate biopsy. Investig Clin Urol 2016;57:94-99.

4. Cheung C, Patel HD, Landis P, Carter HB, Han M. Targeted antimicrobial prophylaxis for transrectal ultrasound-guided prostate biopsy during active surveillance: effect on hospitalization. Urol Oncol 2018;36:158 e7-e12.

5. Wolf JS, Jr., Bennett CJ, Dmochowski RR, et al. Best practice policy statement on urologic surgery antimicrobial prophylaxis. American Urological Association website. https://auanet.org/education/guidelines/antimicrobialprophylaxis.cfm. Published 2014. Accessed April 19, 2021.

6. Bouchillon SK, Badal RE, Hoban DJ, Hawser SP. Antimicrobial susceptibility of inpatient urinary tract isolates of gram-negative bacilli in the United States: 
results from the study for monitoring antimicrobial resistance trends (SMART) program: 2009-2011. Clin Ther 2013;35:872-877.

7. Atalay HA, Canat L, Alkan I, Cakir SS, Altunrende F. Prostate-specific antigen reduction after empiric antibiotic treatment does not rule out biopsy in patients with lower urinary tract symptoms: prospective, controlled, singlecenter study. Prostate Int 2017;5:59-64.

8. Eggener SE, Large MC, Gerber GS, et al. Empiric antibiotics for an elevated prostate-specific antigen (PSA) level: a randomised, prospective, controlled multi-institutional trial. BJU Int 2013;112:925-929.

\title{
Surveillance of hospital water systems to help predict the risk of nosocomial legionellosis-Consider other factors
}

\author{
Hilary Humphreys MD, DSc ${ }^{1,2}$, Peter O'Donovan MBS ${ }^{3}$ and Sharon Dwyer $\mathrm{MSc}^{4}$ \\ ${ }^{1}$ Department of Clinical Microbiology, the Royal College of Surgeons in Ireland, Dublin, Ireland, ${ }^{2}$ Department of Microbiology, Beaumont Hospital, Dublin, Ireland, \\ ${ }^{3}$ Department of Estates and Engineering and Facility Management, Beaumont Hospital, Dublin, Ireland and ${ }^{4}$ Department of Quality and Patient Safety, \\ Beaumont Hospital, Dublin, Ireland
}

To the Editor-We read with interest the study by Nagy et $\mathrm{al}^{1}$ in which they used a statistical fit test to support recommendations on the proportion of positive Legionella tests to indicate a wellmaintained or a well-treated water distribution system. They concluded that surveillance showing a rate of $\leq 30 \%$ water samples positive for Legionella spp provided some reassurance. However, some consideration needs to be given to the healthcare facility, the proportion of at-risk patients and the laboratory diagnosis of clinical cases. ${ }^{2}$ Furthermore, there may be a role for considering how heavy the bioburden is in positive water outlet samples and even possibly the Legionella spp.

Beaumont Hospital is a tertiary-care referral center in Ireland with national specialties in renal transplantation, neurosurgery, cochlear implantation, and several other complex specialties. We have an ongoing multidisciplinary team that oversees and delivers a legionellosis prevention program that includes surveillance and the maintenance of a complex hospital facility that is $>30$ years old. This surveillance includes a range of engineering controls (eg, active review of pipework and removal of dead legs); barcoding of assets; active cloud-based temperature checks on the water system; a comprehensive flushing program; and a blended approach to chemical water treatment with chlorine dioxide, copper silver, and anolyte (a positively charged form of chlorine). Our water outlet sampling regimen includes taking 160 samples per month and following up on any positive outlet to optimize operational conditions with retesting until negative. We treat individual water outlets even if the counts are low and whatever the Legionella spp because of the increasingly complex case mix in our hospital. In recent years, we have seen the proportion of water samples positive for any Legionella spp decline from $>30 \%$ to $<2.5 \%$.

European guidelines are very general in terms of sampling; they recommend "periodic sampling and testing for the presence of Legionella based on risk assessment." 3 They further recommend that the sensitivity of laboratory methods should be capable

\footnotetext{
Author for correspondence: Hilary Humphreys, E-mail: hhumphreys@rcsi.ie

Cite this article: Humphreys H, O’Donovan P, and Dwyer S. (2022). Surveillance of hospital water systems to help predict the risk of nosocomial legionellosis-Consider other factors. Infection Control \& Hospital Epidemiology, 43: 1080-1081, https:// doi.org/10.1017/ice.2021.173
}

of detecting counts of $100 \mathrm{CFU} / \mathrm{L}$ or less but that the results should be appropriately interpreted. ${ }^{3}$ Irish guidelines are more specific and include recommendations on sample types, laboratory analysis and action to be taken if an outlet is positive (eg, if $>1,000 \mathrm{CFU} / \mathrm{L}$ ), control measures, and disinfection of the positive water plus resampling. ${ }^{4}$ However, they do emphasize that "Sampling is not a substitute for good maintenance practices and water treatment." 4

Stout et $\mathrm{al}^{5}$ originally identified the correlation between cases of nosocomial legionellosis and $\geq 30 \%$ of outlets being positive. It seems plausible to assume that the more outlets that are positive, the greater the likelihood of nosocomial legionellosis. However, like most infections, healthcare-acquired legionellosis is multifactorial and will be influenced by the vulnerability or susceptibility of the patient (eg, underlying immunosuppression), how the patient is exposed to water (ie, via aerosols), potentially how heavy the bioburden is in the water outlet, and the species. Although we have traditionally regarded Legionella pneumophilia (especially serogroup 1) as the most pathogenic, other serogroups and species may potentially infect susceptible patients, and some general medical and surgical patients are increasingly on biological agents (eg, for inflammatory bowel disease).

Although we do not disagree with the suggestions and recommendations from Nagy et al, we urge caution in overly relying on the proportion of water outlets positive for Legionella as indicating a relatively lower risk of legionellosis. However, further studies are required to determine the influence of case mix, the number and frequency of surveillance samples taken, where they are taken (eg, the clinical area and water outlet such as shower versus hand-wash basin), existing and ongoing preventative measures, and both the bioburden and Legionella species.

Acknowledgments. We acknowledge the support and input of staff from many disciplines and departments who help prevent nosocomial legionellosis and other infections.

Financial support. The collation of material and the preparation of this manuscript was not supported by external funding but was undertaken during routine clinical and academic activities.

Conflicts of interest. H.H. has received research monies and a consultancy fee from Astellas (Ireland) and Pfizer (Ireland) during the last 4 years but not in areas related to the contents of this manuscript. None of the other authors has a conflict of interest to declare. 